\title{
Viscoelastometry for detecting oral anticoagulants
}

\author{
Philipp Groene ${ }^{1^{*}}$ D, Daniela Wagner ${ }^{1}$, Tobias Kammerer ${ }^{1,2}$, Lars Kellert ${ }^{3}$, Andreas Giebl $^{4}$, Steffen Massberg ${ }^{5}$ and \\ Simon Thomas Schäfer ${ }^{1}$
}

\begin{abstract}
Background: Determination of anticoagulant therapy is of pronounced interest in emergency situations. However, routine tests do not provide sufficient insight. This study was performed to investigate the impact of anticoagulants on the results of viscoelastometric assays using the ClotPro device.

Methods: This prospective, observational study was conducted in patients receiving dabigatran, factor Xa (FXa)inhibitors, phenprocoumon, low molecular weight heparin (LMWH) or unfractionated heparin (UFH) (local ethics committee approval number: 17-525-4). Healthy volunteers served as controls. Viscoelastometric assays were performed, including the extrinsic test (EX-test), intrinsic test (IN-test) Russel's viper venom test (RW-test), ecarin test (ECA-test), and the tissue plasminogen activator test (TPA-test).

Results: 70 patients and 10 healthy volunteers were recruited. Clotting time in the EX-test ( $\left.C T_{\text {EX-test }}\right)$ was significantly prolonged versus controls by dabigatran, FXa inhibitors and phenprocoumon. $C_{1 N \text {-test }}$ was prolonged by dabigatran, FXa inhibitors and UFH. Dabigatran, FXa inhibitors and UFH significantly prolonged $\mathrm{CT}_{\mathrm{RW} \text {-test }}$ in comparison with controls (median 200, 207 and 289 vs 63 s, respectively; all $p<0.0005$ ). Only dabigatran elicited a significant increase in $\mathrm{CT}_{\mathrm{ECA} \text {-test }}$ compared to controls (median 307 vs $73 \mathrm{~s} ; p<0.0001$ ). $\mathrm{CT}_{\mathrm{ECA} \text {-test }}$ Correlated strongly with dabigatran plasma concentration (measured by anti-lla activity; $r=0.9970 ; p<0.0001$ ) and provided $100 \%$ sensitivity and 100\% specificity for detecting dabigatran. Plasma concentrations (anti-XA activity) of FXa inhibitors correlated with $\mathrm{CT}_{\text {RW-test }}(r=0.7998$; $p<0.0001$ ), and $\mathrm{CT}_{\mathrm{RW} \text {-test }}$ provided $83 \%$ sensitivity and 64\% specificity for detecting FXa inhibitors.
\end{abstract}

Conclusions: In emergency situations, ClotPro viscoelastometric assessment of whole-blood samples may help towards determining the presence and type of anticoagulant class that a patient is taking.

Trial registration: German clinical trials database ID: DRKS00015302.

Keywords: Anticoagulants, Coagulation tests, Dabigatran, Factor Xa (FXa) inhibitors, Heparin, Viscoelastometry

\section{Background}

Patients with pre-existing cardiovascular disease are at increased risk of thromboembolic events. Considering the trend towards increased longevity among human populations across the world, the use of anticoagulation therapy has become widespread $[1,2]$. A range of

\footnotetext{
* Correspondence: philipp.groene@med.uni-muenchen.de

'Department of Anaesthesiology, University Hospital Munich, LMU Munich, Marchioninistraße 15, 81377 Munich, Germany

Full list of author information is available at the end of the article
}

effective treatments are available, but patients receiving anticoagulation therapy can be at risk of excessive bleeding, e.g. in trauma or emergency surgery [3-5]. These situations may require immediate treatment of anticoagulant effects, whereas interventions differ between anticoagulants $[6,7]$. Depending on urgency, vitamin $K$ antagonists (VKAs, e.g. warfarin) may be reversed using vitamin $\mathrm{K}$ or prothrombin complex concentrates (PCCs) $[8,9]$. Furthermore, specific reversal agents have been developed for direct oral anticoagulants (DOACs),

(c) The Author(s). 2021 Open Access This article is licensed under a Creative Commons Attribution 4.0 International License, which permits use, sharing, adaptation, distribution and reproduction in any medium or format, as long as you give appropriate credit to the original author(s) and the source, provide a link to the Creative Commons licence, and indicate if changes were made. The images or other third party material in this article are included in the article's Creative Commons licence, unless indicated otherwise in a credit line to the material. If material is not included in the article's Creative Commons licence and your intended use is not permitted by statutory regulation or exceeds the permitted use, you will need to obtain permission directly from the copyright holder. To view a copy of this licence, visit http://creativecommons.org/licenses/by/4.0/. The Creative Commons Public Domain Dedication waiver (http://creativecommons.org/publicdomain/zero/1.0/) applies to the data made available in this article, unless otherwise stated in a credit line to the data. 
including idarucizumab for direct thrombin inhibitors and andexanet alfa for emergency bleeding situations in patients on direct FXa- antagonists $[9,10]$.

Thus, in an unknown patient with emergency bleeding, it may be necessary to determine the presence and type of anticoagulant therapy by performing coagulation tests. Fast, reliable detection and differentiation are essential in patients with severe bleeding, to enable administration of the most effective reversal therapy (e.g. antagonization by specific antidotes such as andexanet alfa or idarucizumab). The results of standard laboratory tests (e.g. international normalized ratio [INR], activated partial thromboplastin time [aPTT]), as well as standard viscoelastic tests (e.g. EXTEM clotting time (CT), TEG R-time), are affected by anticoagulant drugs [11-14]. However, these routine tests do not readily provide sufficient insight to determine exactly which drug a patient is taking. Anti-factor Xa and antifactor IIa tests may help determine presence of a factor $\mathrm{Xa}$ inhibitor or a direct thrombin inhibitor but these laboratory assessments are affected by heparin and they have prolonged turnaround times. There is a need for specific diagnostic tools suitable for point-of-care use in emergency situations $[15,16]$.

A newly developed viscoelastometric device (ClotPro, enicor $\mathrm{GmbH}$, Munich, Germany) using different methodology from ROTEM or TEG, provides rapid bedside evaluation of whole-blood coagulation [17, 18]. Multiple assays can be performed concurrently as the device has six test channels [17]. Some assays are comparable with commonly used ROTEM/TEG assays (e.g. extrinsic test [EX-test], functional fibrinogen test [FIB-test], intrinsic test [IN-test]). Additional assays have been developed specifically for the detection and differentiation of anticoagulants: the Russel's viper venom test (RVV-test) and the ecarin test (ECA-test). We performed a trial to assess the effects of anticoagulant drugs, including DOACs, on viscoelastometric test results.

\section{Methods}

This in vivo, prospective, observational, single-center study was conducted between November 2018 and October 2019. It was registered at the German clinical trials database (ID: DRKS00015302), and the protocol was approved by the Ludwig-Maximilians-University ethics committee (No 17-525-4). The study was performed in accordance with the Declaration of Helsinki, with written informed consent being obtained from all participants before they undertook any of the study procedures.

Adults who had been treated with any one of the following drugs for at least 7 days were eligible to participate: dabigatran, rivaroxaban, apixaban, edoxaban, phenprocoumon, low molecular weight heparin
(LMWH) or unfractionated heparin (UFH). In each of these seven treatment groups, concurrent treatment with platelet inhibitors (e.g. acetylsalicylic acid or clopidogrel) was permissible. A control group of healthy volunteers was recruited, bringing the total number of study groups to eight. Exclusion criteria were as follows: age $<18$ years, treatment with more than one anticoagulant, coagulation disorders (e.g. von Willebrand's disease), and myelodysplastic syndrome.

Each study participant provided a single blood sample for analysis. To reflect the clinical circumstances of patients in whom emergency bleeding may occur at any time, no measures were taken to control the time at which samples were taken.

All samples were tested using a ClotPro device (enicor $\mathrm{GmbH}$, Munich, Germany) within $2 \mathrm{~h}$ after blood withdrawal, under standardized conditions $\left(37^{\circ} \mathrm{C}\right.$, maximum test run time $4.500 \mathrm{~s})$. Five assays were performed on each sample (EX-test, IN-test, ECA-test, RVV-test and tissue plasminogen activator test [TPA-test]), and the following variables were documented: $\mathrm{CT}$, clot formation time (CFT), clot amplitude at 5 and $10 \mathrm{~min}$ after CT (A5, A10), maximum clot firmness (MCF), maximum lysis (ML), as well as lysis time (LT; time from CT until $50 \%$ lysis) and lysis onset time (LOT; time from CT until $15 \%$ lysis) for TPA-test. Standard laboratory tests were performed on each sample: INR (Thromborel S; Siemens Healthcare $\mathrm{GmbH}$, Erlangen, Germany), thrombin time (TT) (Berichrom Thrombinreagenz; Siemens Healthcare GmbH, Erlangen, Germany), aPTT (Actin FSL; Siemens Healthcare $\mathrm{GmbH}$, Erlangen, Germany) and full blood count. Anti-activated factor X (anti-Xa) and antiactivated factor II (anti-IIa) tests were also performed, using Biophen Heparin LRT 7.5 (Hyphen Biomed, Neuville-sur-Oise, France).

Quality controls were performed according to institutional and manufacturers standards.

\section{Statistical considerations}

10 individuals per study group were recruited $(N=80)$. This sample size was calculated from one-way analysis of variance of the magnitude of expected differences in CT between the oral anticoagulants included in the study and healthy controls. A large difference between the anticoagulants and healthy controls was assumed (effect size $f=0.4$; alpha error 0.05 ; power 0.8 ). The software used was G*Power 3.1 (provided by the University of Düsseldorf). This procedure was done due to preliminary work on in-vitro dose-effect curves with DOACs and the new tests.

Statistical analyses of the results were performed using Graph Pad Prism 8 (La Jolla, USA) and SPSS Version 25 (IBM, Armonk, USA). All data are presented as median with interquartile range (IQR; 25th-75th percentile) 
unless indicated otherwise. For statistical comparison of study groups, results from the three activated factor $\mathrm{X}$ (FXa) inhibitors were pooled. Differences between groups were analyzed using the Kruskal-Wallis-test and Dunn's multiple comparison test. The significance level (alpha) was adjusted for multiple testing $(p=0 \cdot 05 / \mathrm{n})$. Associations between variables were assessed using Spearman's correlation coefficient.

To investigate the detection of an anticoagulant drug, all samples from patients who had taken the drug were compared against samples from controls. Receiver operating characteristic (ROC) analyses were performed to evaluate the sensitivity and specificity of test parameters for detecting anticoagulant drugs. Optimal cut-off values were determined by Youden's Index.

\section{Results}

A total of 80 individuals were recruited for the study, and their characteristics are shown in Table 1 . The last intake of anticoagulant medication was at a median of $3.75 \mathrm{~h}$ (range, 1.00-12.00) before taking the blood sample in patients treated with dabigatran, $6.00 \mathrm{~h}$ (range,
0.50-17.50) among those receiving FXa inhibitors, and $3.25 \mathrm{~h}$ (range $0.50-13.50$ ) in patients on LMWH.

Median $\mathrm{CT}_{\text {EX-test }}$ was $44 \mathrm{~s}$ (IQR 38-47 s) in the control group (see Fig. 1a, Supplemental Tables 1A and B). Significant prolongation of $\mathrm{CT}_{\mathrm{EX} \text {-test }}$ was observed with several of the anticoagulants: dabigatran, FXa inhibitors except for apixaban, and phenprocoumon. Dabigatran produced the largest increase in $\mathrm{CT}_{\mathrm{EX} \text {-test }}$ : median value $155 \mathrm{~s}$ (IQR 96-206 s); $p<0.0001$ versus controls. $\mathrm{CT}_{\mathrm{EX}-\mathrm{t}-}$ est was not significantly prolonged versus controls by UFH or LMWH. None of the anticoagulant drugs produced statistically significant changes in $\mathrm{A} 5_{\mathrm{EX} \text {-test }}$ or $\mathrm{MCF}_{\mathrm{EX} \text {-test }}$ compared to the control group (Supplemental Table $1 \mathrm{~A})$. In the ROC analysis, the optimal $\mathrm{CT}_{\mathrm{EX} \text {-test }}$ cut-off for FXa-inhibitor detection was determined as $69 \mathrm{~s}$. This cut-off was associated with an AUC of 0.649 (standard error [SE], 0.062), sensitivity of 70\%, and specificity of $64 \%$.

In the control group, the median $\mathrm{CT}_{\text {IN-test }}$ was $142 \mathrm{~s}$ (IQR 135-148 s; Fig. 1b, Supplemental Tables 1A and B). Statistically significant prolongation of $\mathrm{CT}_{\mathrm{IN} \text {-test }}$ was seen with dabigatran and FXa inhibitors except for

Table 1 Demographics and other characteristics of the study participants. Data are presented as median (interquartile range), unless indicated otherwise

\begin{tabular}{|c|c|c|c|c|c|c|c|c|}
\hline & $\begin{array}{l}\text { Controls } \\
(n=10)\end{array}$ & $\begin{array}{l}\text { Rivaroxaban } \\
(n=10)\end{array}$ & $\begin{array}{l}\text { Apixaban } \\
(n=10)\end{array}$ & $\begin{array}{l}\text { Edoxaban } \\
(n=10)\end{array}$ & $\begin{array}{l}\text { Dabigatran } \\
(n=10)\end{array}$ & $\begin{array}{l}\text { Phenprocoumon } \\
(n=10)\end{array}$ & $\begin{array}{l}\text { LMWH } \\
(n=10)\end{array}$ & $\begin{array}{l}\text { UFH }(n= \\
10)\end{array}$ \\
\hline $\begin{array}{l}\text { Female/male } \\
\text { gender, } \mathrm{n}(\%)\end{array}$ & $6 / 4(60 / 40)$ & $5 / 5(50 / 50)$ & $8 / 2(80 / 20)$ & $9 / 1(90 / 10)$ & $8 / 2(80 / 20)$ & $6 / 4(60 / 40)$ & $6 / 4(60 / 40)$ & $3 / 7(30 / 70)$ \\
\hline Age (years) & $60(58-64)$ & $71(54-81)$ & $82(79-87)$ & $77(73-83)$ & $72(58-79)$ & $82(45-86)$ & $70(42-79)$ & $74(63-82)$ \\
\hline BMI $\left(\mathrm{kg} / \mathrm{m}^{2}\right)$ & $\begin{array}{l}27.1(25.6- \\
30.7)\end{array}$ & $23.7(22.0-31.7)$ & $\begin{array}{l}23.9(23.2- \\
26.0)\end{array}$ & $\begin{array}{l}27.0(24.0- \\
29.2)\end{array}$ & $\begin{array}{l}26.3(23.6- \\
29.5)\end{array}$ & $26.6(24.6-30.2)$ & $\begin{array}{l}24.3(20.3- \\
28.1)\end{array}$ & $\begin{array}{l}26.6(22.0- \\
27.9)\end{array}$ \\
\hline \multicolumn{9}{|l|}{ ASA status, n (\%) } \\
\hline 1 & $2(20)$ & 0 & 0 & 0 & 0 & 0 & 0 & 0 \\
\hline 2 & $8(80)$ & $2(20)$ & 0 & 0 & $3(30)$ & 0 & $1(10)$ & 0 \\
\hline 3 & 0 & $8(80)$ & $10(100)$ & $10(100)$ & $7(70)$ & $10(100)$ & $9(90)$ & $9(90)$ \\
\hline 4 & 0 & 0 & 0 & 0 & 0 & 0 & 0 & $1(10)$ \\
\hline \multicolumn{9}{|l|}{ Diabetes, n (\%) } \\
\hline Yes & 0 & $2(20)$ & $2(20)$ & $2(20)$ & $2(20)$ & $4(40)$ & $1(10)$ & $3(30)$ \\
\hline No & $10(100)$ & $8(80)$ & $8(80)$ & $8(80)$ & $8(80)$ & $6(60)$ & $9(90)$ & $6(70)$ \\
\hline Creatinine (mg/dL) & $\begin{array}{l}0.9(0.78- \\
0.95)\end{array}$ & $1.0(0.89-1.49)$ & $1.2(1.2-1.4)$ & $1.21 .1-1.4)$ & $1.1(0.9-1.3)$ & $1.5(1.1-2.6)$ & $\begin{array}{l}1.1(0.88- \\
1.4)\end{array}$ & $\begin{array}{l}1.0(0.6- \\
2.5)\end{array}$ \\
\hline GFR (ml/min) & $96(93-106)$ & 79 (65-99) & $56(48-82)$ & $71(52-81)$ & $74(64-104)$ & $61(35-84)$ & $\begin{array}{l}92(70- \\
112)\end{array}$ & $73(23-86)$ \\
\hline Platelets ( $\left.10^{9} / \mathrm{L}\right)$ & $\begin{array}{l}262(210- \\
289)\end{array}$ & $214(141-265)$ & $\begin{array}{l}247(159- \\
294)\end{array}$ & $220(180-271)$ & $229(184-293)$ & $239(170-266)$ & $\begin{array}{l}282(211- \\
330)\end{array}$ & $\begin{array}{l}227(156- \\
350)\end{array}$ \\
\hline $\mathrm{Hb}(\mathrm{mg} / \mathrm{dL})$ & $\begin{array}{l}14.3(13.7- \\
15.2)\end{array}$ & $12.5(10.0-14.6)$ & $\begin{array}{l}11.8(9.9- \\
13.6)\end{array}$ & $\begin{array}{l}13.1(11.9- \\
14.1)\end{array}$ & $\begin{array}{l}13.6(11.4- \\
15.1)\end{array}$ & $12.2(10.7-15.3)$ & $\begin{array}{l}11.0(9.4- \\
12.6)\end{array}$ & $\begin{array}{l}8.3(7.4- \\
9.1)\end{array}$ \\
\hline Hematocrit & $\begin{array}{l}0.41(0.39- \\
0.43)\end{array}$ & $0.38(0.31-0.43)$ & $\begin{array}{l}0.36(0.3- \\
0.41)\end{array}$ & $\begin{array}{l}0.38(0.37- \\
0.41)\end{array}$ & $\begin{array}{l}0.40(0.34- \\
0.44)\end{array}$ & $0.36(0.32-0.45)$ & $\begin{array}{l}0.31(0.28- \\
0.35)\end{array}$ & $\begin{array}{l}0.26(0.22- \\
0.28)\end{array}$ \\
\hline Fibrinogen (mg/dL) & $\begin{array}{l}367(325- \\
411)\end{array}$ & 413 (359-447) & $\begin{array}{l}530(366- \\
594)\end{array}$ & $481(310-570)$ & $365(298-425)$ & $420(390-500)$ & $\begin{array}{l}429(341- \\
597)\end{array}$ & $\begin{array}{l}414(319- \\
509)\end{array}$ \\
\hline
\end{tabular}

ASA American society of anesthesiologist status; BMI body mass index; GFR glomerular filtration rate; $H b$ hemoglobin; $L M W H$ low molecular weight heparin; UFH unfractionated heparin 


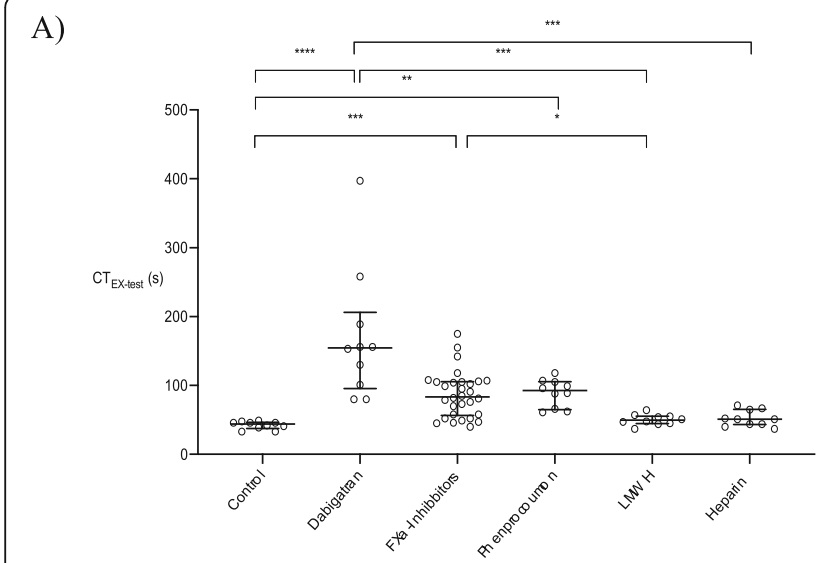

B)

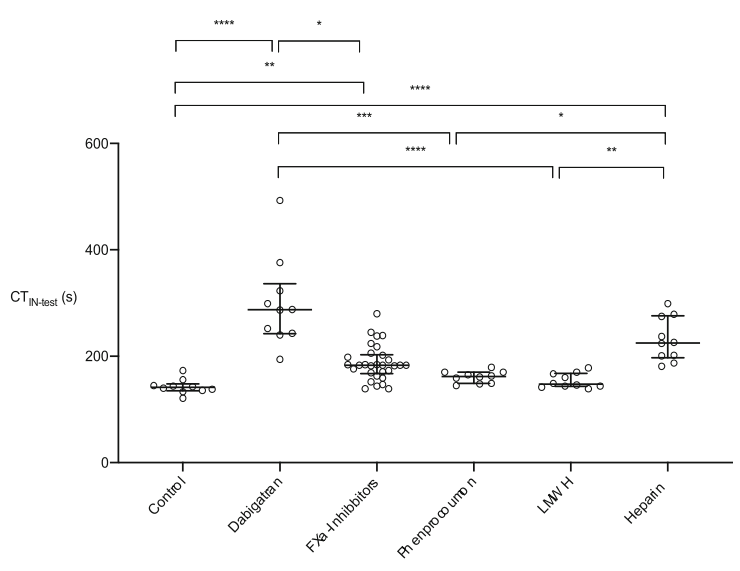

C)

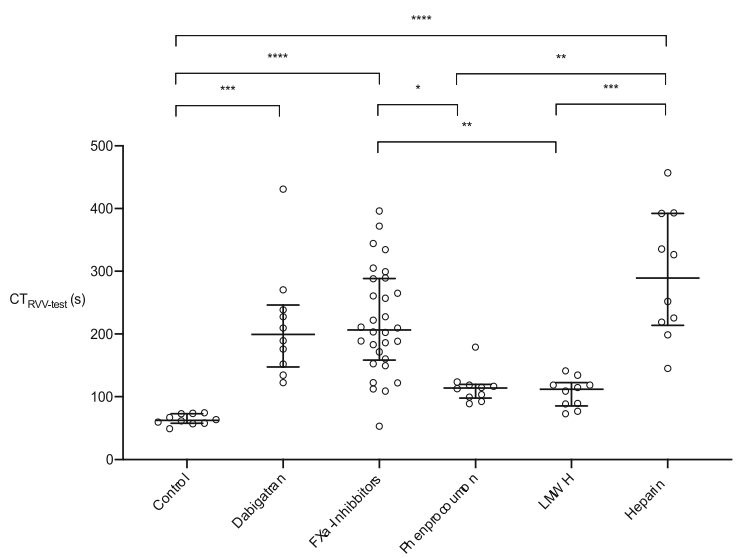

D)

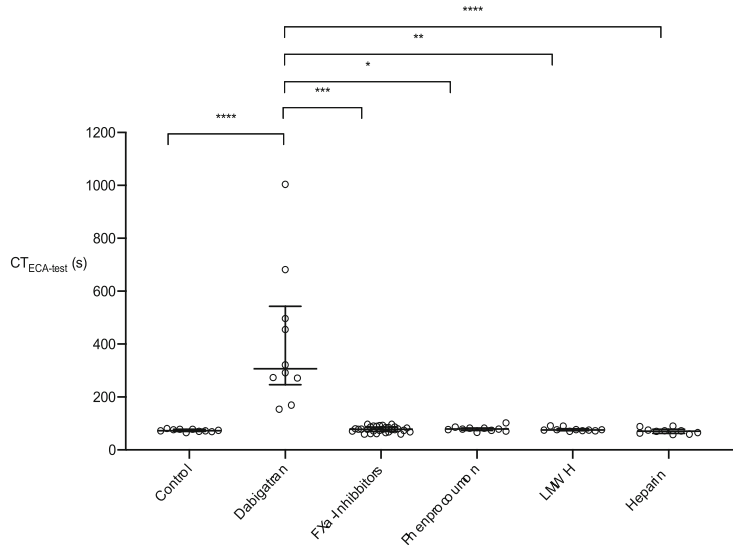

Fig. 1 Viscoelastometric CT results (scatter dot plots) from the control group and the anticoagulant groups. Data are shown as median and interquartile range. a EX-test, $\mathbf{b} \mid \mathrm{N}$-test, $\mathbf{c}$ RW-test and $\mathbf{d}$ ECA-test. ${ }^{*} \mathrm{p}<0.05,{ }^{* *} p<0.01,{ }^{* * *} p<0.001,{ }^{* * * *} p<0.0001$. CT, clotting time; LMWH, low molecular weight heparin

apixaban. Unlike with $\mathrm{CT}_{\mathrm{EX} \text {-test }}$ UFH also produced significant prolongation of $\mathrm{CT}_{\text {IN-test. }}$ The optimal $\mathrm{CT}_{\mathrm{IN} \text {-test }}$ cut-off for UFH was determined as $186 \mathrm{~s}$. This cut-off was associated with an area under the curve (AUC) of 0.768 (SE 0.056) and a sensitivity of $90 \%$ and a specificity of $67 \%$.

$\mathrm{CT}_{\mathrm{RVV} \text {-test }}$ in controls was $63 \mathrm{~s}$ (IQR $58-73 \mathrm{~s}$; Fig. 1c, Supplemental Tables 1A and B). Significantly increased values for this parameter were observed with UFH, FXa inhibitors and dabigatran. The largest increase in $\mathrm{CT}_{\mathrm{RVV-test}}$ was observed with UFH (median 289 [214392] seconds; $p<0.0001$ versus controls). Correlations were observed between $\mathrm{CT}_{\mathrm{RVV} \text {-test }}$, and plasma FXainhibitor concentrations measured by anti-Xa activity (rivaroxaban: $\mathrm{r}=0.5636, p=0.0963$; apixaban: $\mathrm{r}=0.5714$, $p=0.2$ ), and statistical significance was reached with edoxaban $(\mathrm{r}=0.9394 ; p=0.0002$ and pooled FXa inhibitors: $\mathrm{r}=0.7998 ; \mathrm{p}<0.0001) . \mathrm{CT}_{\mathrm{RVV} \text {-test }}$ also showed statistically significant correlation with anti-Xa activity of samples from patients in the LMWH group $(r=0.6687$; $p=0.0395)$. In the ROC analysis, the optimal $\mathrm{CT}_{\mathrm{RVV} \text {-test }}$ cut-off for FXa-inhibitor detection was determined as $147 \mathrm{~s}$. This cut-off was associated with an AUC of 0.721 (standard error [SE], 0.058), sensitivity of $83 \%$, and specificity of $64 \%$. T Detection of apixaban (apixaban vs. controls) was improved using RVV-test (90\% sensitivity and $100 \%$ specificity) compared to EX-test (80\% sensitivity and $80 \%$ specificity).

The optimal $\mathrm{CT}_{\mathrm{RVV} \text {-test }}$ cut-off for UFH was determined as 194 s. This cut-off was associated with an AUC of 0.808 (SE 0.064 ) and a sensitivity of $90 \%$ and a specificity of $63 \%$.

Dabigatran was the only anticoagulant to produce a significant change versus control in $\mathrm{CT}_{\mathrm{ECA} \text {-test }}$ (median 307 [246-543] vs 73 [70-78] seconds, respectively; $p<$ 0.0001 Fig. 1d, Supplemental Tables 1A and B). Dabigatran plasma concentrations (measured by anti-IIa activity) were strongly correlated with $\mathrm{CT}_{\mathrm{ECA} \text {-test }}(\mathrm{r}=0.9970$; $\mathrm{p}<0.0001)$. The optimal cut-off value of $\mathrm{CT}_{\mathrm{ECA} \text {-test }}$ for detecting dabigatran was calculated as $128 \mathrm{~s}$. This 
yielded an AUC for the ROC curve of 1.00 (SE, 0.00), with $100 \%$ sensitivity and $100 \%$ specificity.

In all eight study groups, median values for $\mathrm{CT}_{\text {TPA-test }}$ were numerically comparable to those for $\mathrm{CT}_{\mathrm{EX} \text {-test. }}$ Accordingly, as with $\mathrm{CT}_{\mathrm{EX} \text {-test }}, \mathrm{CT}_{\mathrm{TPA} \text {-test }}$ was significantly prolonged versus controls by dabigatran, FXa inhibitors and phenprocoumon (Fig. 2a, Supplemental Tables 1A and $\mathrm{B}$ ). Only dabigatran had a notable effect on lysis in the TPA-test (Fig. 2b-d; Supplemental Table 1A). The effect of dabigatran was most pronounced with LOT, where the median value of $52 \mathrm{~s}$ (IQR 40-63 s) was significantly shorter than that of controls (99 [83-101] seconds; $p=0.0086$ ).

Standard laboratory test results are summarized in Supplementary Table 1A. aPTT was significantly increased by UFH and dabigatran versus controls $(p<$ 0.0001 for both comparisons). Phenprocoumon was the only anticoagulant with significant impact on INR versus controls $(\mathrm{p}<0.0001)$. Compared with the control group (median 17 [IQR 17-18] seconds), TT was significantly prolonged by dabigatran (144 [114-150] seconds; $p=$ 0.0003). Statistically significant prolongation of TT was also observed with UFH (48 s $(38-123 ; p=0.004)$, although the extent of this prolongation with UFH was much less than that with dabigatran.

\section{Discussion}

This study demonstrates that viscoelastometry is sensitive to a range of anticoagulants, including DOACs. The effects of anticoagulants on the 'conventional' EX-test are not specific, while novel parameters (e.g., CT in the RVV-test, CT in the ECA-text, LOT in the TPA-test) could be helpful in differentiating between DOAC classes (direct thrombin inhibitors and FXa-inhibitors).

The direct thrombin inhibitor dabigatran significantly prolonged $\mathrm{CT}$ in all five assays compared to controls. Of note, this was the only drug which prolonged $\mathrm{CT}_{\mathrm{ECA} \text {-test }}$, and it was also the only drug which shortened LOT in the TPA-test. FXa inhibitors prolonged CT in all assays except the ECA-test. The magnitude of effect of the FXa
A)

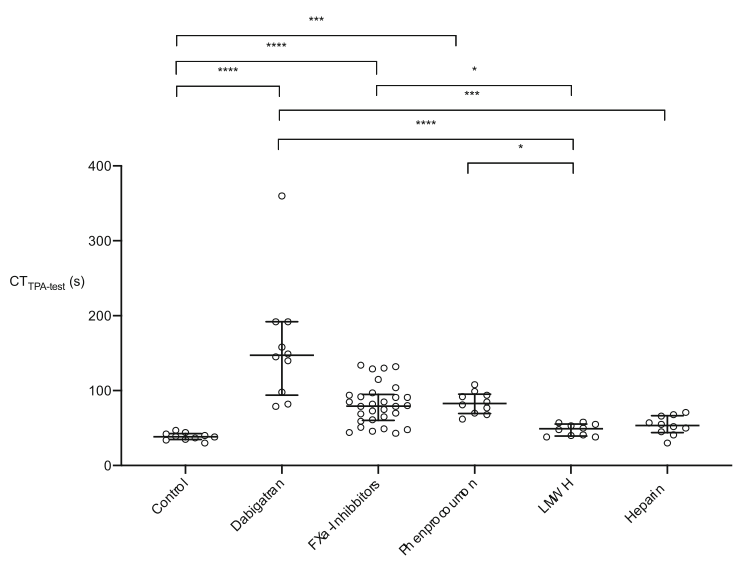

C)

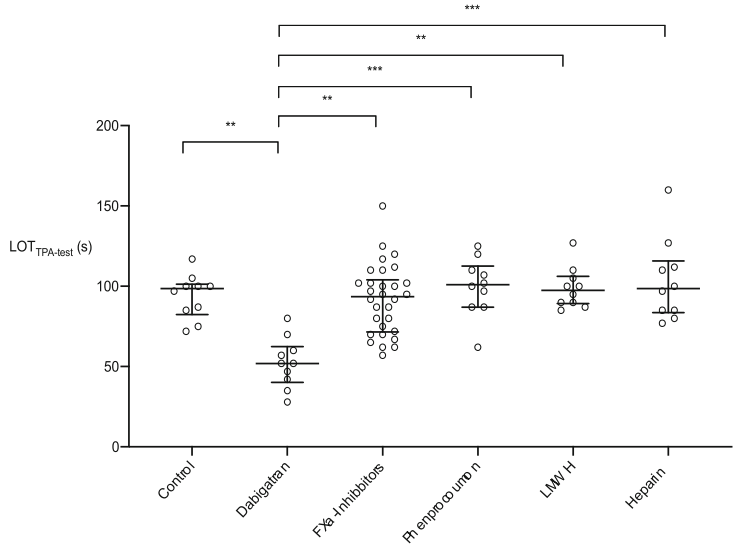

B)

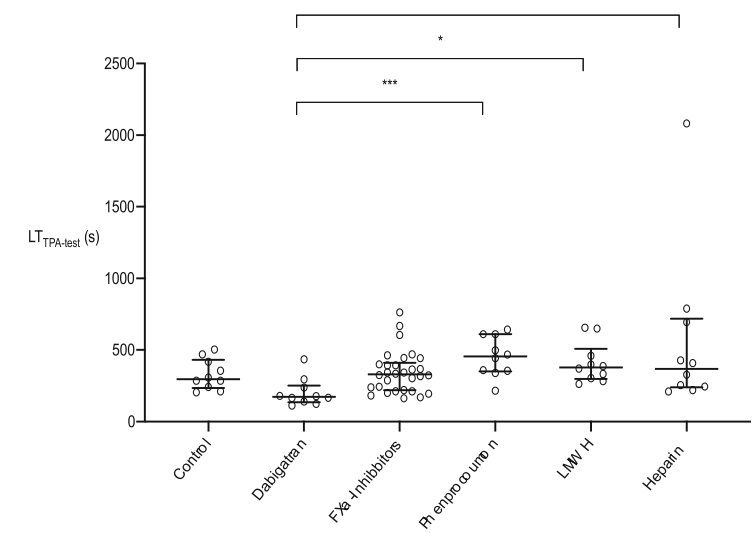

D)

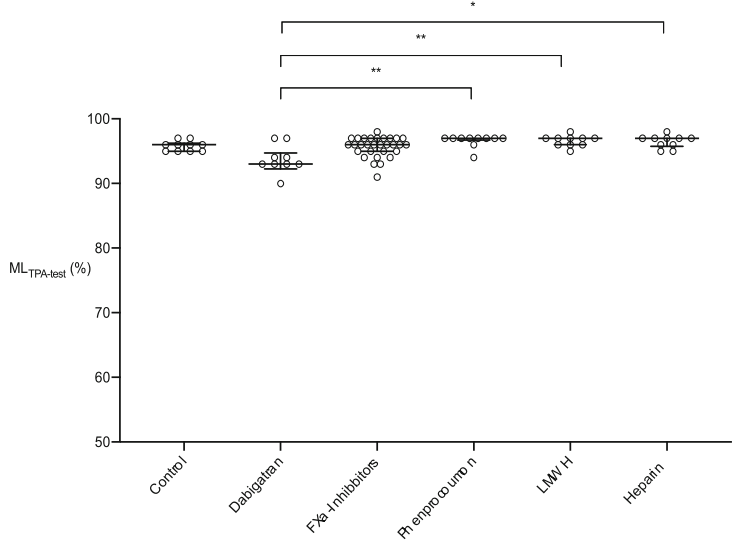

Fig. 2 Viscoelastometric TPA-test results (scatter dot plots) from the control group and the anticoagulant groups. Data are shown as median and interquartile range. a CT, b LT, $\mathbf{c} L O T$ and $\mathbf{d} \mathrm{ML}$. ${ }^{*} p<0.05,{ }^{* *} p<0.01,{ }^{* * *} p<0.001,{ }^{* * * *} p<0.0001$. CT, clotting time; LT, lysis time; LOT, lysis onset time; ML, maximum lysis; LMWH, low molecular weight heparin 
inhibitors on $\mathrm{CT}$ was less than dabigatran, except in the RVV-test where the median CT was similar in both the dabigatran and FXa-inhibitor groups. Compared with controls, phenprocoumon significantly prolonged $\mathrm{CT}$ in the TPA-test and the EX-test, UFH significantly prolonged CT in the IN-test and the RVV-test, and LMWH did not significantly prolong CT in any of the assays.

Correlations observed in this study show that viscoelastometry by ClotPro is suitable for detecting a range of anticoagulants. We observed a strong correlation between $\mathrm{CT}_{\mathrm{ECA} \text {-test }}$ and anti-IIa activity (a surrogate for the concentration of dabigatran). It enabled the calculation of a threshold $\mathrm{CT}_{\mathrm{ECA} \text {-test }}$ value for detecting the presence of dabigatran, and the associated sensitivity and specificity values of $100 \%$ show possible capabilities in daily practice. Reliable quantification of dabigatran levels also appears possible, subject to appropriate calibration of $\mathrm{CT}_{\mathrm{ECA} \text {-test }}$. These data are in accordance with our earlier study using the ROTEM delta thromboelastometry device, showing that an ecarin-based test is highly specific and sensitive to direct thrombin inhibitors [19]. Correlation was also observed between $\mathrm{CT}_{\mathrm{RVV} \text {-test }}$ and anti-Xa activity (a surrogate for the concentration of FXa inhibitor or heparin). Further studies are planned to confirm the potential of $\mathrm{CT}_{\mathrm{RVV} \text {-test }}$ for quantifying $\mathrm{FXa}$-inhibitor and LMWH plasma concentrations.

The results of this study suggest that a combination of standard and newly developed viscoelastometric tests like the RVV-test and the ECA-test may provide benefits compared to conventional, standard viscoelastic coagulation monitoring for detection of and differentiation between DOACs $[14,20,21]$. High values for $\mathrm{CT}_{\text {ECA-test }}$ and $\mathrm{CT}_{\mathrm{RVV} \text {-test }}$ enable confident deduction that a patient is receiving dabigatran (or perhaps a different direct thrombin inhibitor). Prolongation of $\mathrm{CT}_{\mathrm{RVV} \text {-test }}$ but not $\mathrm{CT}_{\mathrm{ECA} \text {-test }}$ is consistent with the patient receiving an FXa inhibitor. However, because $\mathrm{CT}_{\mathrm{RVV} \text {-test }}$ is also prolonged by heparin and (to a lesser extent) by phenprocoumon, it is not clear that the tests performed in this study enable reliable differentiation of FXa inhibitors from other anticoagulant types. Further work is clearly needed in this respect.

In addition to the device used here, there are at least two other devices for point-of-care assessment of DOAC anticoagulation. TEG $6 \mathrm{~s}$ is used with cartridges comprising four channels, each with separate reagents to enable four assays to be run simultaneously. In a study performed using an experimental cartridge, detection and classification of DOACs were reported to be achievable with TEG $6 \mathrm{~s}$ [15]. The experimental cartridge that was used included one channel with ecarin for detecting dabigatran and another channel with FXa for detecting FXa inhibitors. Assays designed specifically for the detection and classification of DOACs have previously been analyzed using the ROTEM device [19]. Our group recently showed the feasibility of this approach for dabigatran and rivaroxaban [19]. In addition to the sensitivity and specificity of the available tests, a range of other factors (e.g. ease of use, time taken to obtain results, costs, availability) are likely to influence which device is adopted in clinical practice. The 'active tip' format of the ClotPro device enables assessments to be performed rapidly with an uncomplicated testing procedure and no requirement for reagent handling by the device user. TEG $6 \mathrm{~S}$ requires pipetting of blood into the cartridge, whereas ROTEM sigma is a fully automated system. The ClotPro test that is most sensitive to FXa inhibitors (the RVVtest) uses a different approach from that studied by our group with the ROTEM device (TFTEM). In the RVVtest, the snake venom from Daboia Russelli (Russel's viper venom) is used to trigger coagulation via $F X$, and this is more specific than the TFTEM assay, which is a diluted EXTEM test. On the other hand, the two ClotPro and ROTEM tests exhibiting high sensitivity to dabigatran (ECA-test and ECATEM) both use ecarin and are therefore similar. In a recently published study by Oberladstätter et al., acutely injured patients on anticoagulant therapy were evaluated using the ClotPro system [22]. The results showed high sensitivity and specificity of the ECA-test for dabigatran and of the RVV-test for FXa inhibitors. The findings of their study are comparable to our results concerning the DOACs in showing the potential application of ClotPro for determining a patient's anticoagulant treatment. In contrast to the study by Oberladstätter et al., we studied the effects of UFH and LMWH on RVV-test results; strong correlation between $\mathrm{CT}_{\mathrm{RVV} \text {-test }}$ and anti-Xa activity was seen with $\mathrm{LMWH}$ and, with UFH, the sensitivity for detection (90\%) was as high as that with the IN-test.

Standard laboratory tests in the present study confirmed the presence of clinically relevant plasma concentrations of the anticoagulants. Dabigatran resulted in significant increases in aPTT and TT, while FXa inhibitors had no significant effect on standard laboratory coagulation tests. Phenprocoumon significantly increased INR and, like dabigatran, UFH produced significant increases in aPTT and TT. These results are consistent with previous observations. It has been reported that $\mathrm{TT}$ is sensitive to dabigatran but not to FXa inhibitors, while the calibrated antiXa test can be used for detection of FXa inhibitors but is not affected by dabigatran [23, 24]. The effects of UFH on aPTT and anti-Xa, and of VKAs such as phenprocoumon on INR, are well known $[25,26]$.

Limitations of the current study include the modest number of patients, questionable robustness of the sample size calculation (the effect size differs between drugs and tests included in the study), and the lack of blinding. Therefore, further data will be needed to confirm our 
results. The allowance for variability in dosing and the time between last intake and blood sampling in this study could be considered as further limitations, due to the resulting variability in plasma drug levels. On the other hand, the real-world approach may be considered as a strength of the study because it means the results are applicable to routine clinical practice. Study participants were not selected according to clinical circumstances and the results should be applicable to all patients receiving the drugs included in our study, regardless of the indication for anticoagulant therapy.

\section{Conclusions}

This study has demonstrated that a combination of standard and newly developed viscoelastometric tests on the ClotPro device enable detection of a range of anticoagulants including DOACs. The ECA-test enables detection of dabigatran with $100 \%$ specificity and sensitivity. Using RVV-test improves detection of FXa-inhibitors but not differentiation. Quantification of some drugs such as edoxaban, LMWH and dabigatran may be possible; further studies are needed to ascertain whether sufficiently accurate calibration can be achieved. Thus, viscoelastometry may potentially be a useful alternative to standard laboratory methods for the detection and differentiation of anticoagulant drugs.

\section{Abbreviations}

A5 / A10: Clot amplitude at 5 and 10 min after CT; Anti-lla: Anti-activated factor II; Anti-Xa: Anti-activated factor X; aPTT: Activated partial thromboplastin time; AUC: Area under the curve; CFT: Clot formation time; CT: Clotting time; DOAC: Direct oral anticoagulant; ECA-test: Ecarin test; EXtest: Extrinsic test; FIB-test: Functional fibrinogen test; IN-test: Intrinsic test; INR: International normalized ratio; IQR: Interquartile range; LMWH: LoW molecular weight heparin; LOT: Lysis onset time; LT: Lysis time;

MCF: Maximum clot firmness; ML: Maximum lysis; PCC: Prothrombin complex concentrate; ROC: Receiver operating characteristic; RW-test: Russel's viper venom test; SE: Standard error; TPA-test: Tissue plasminogen activator test; Tा: Thrombin time; UFH: Unfractionated heparin; VKA: Vitamin K antagonist

\section{Supplementary Information}

The online version contains supplementary material available at https://doi. org/10.1186/s12959-021-00267-w.

Additional file 1 Supplemental Table 1A: Viscoelastometric and standard laboratory test results from the control group and the anticoagulant groups. Data are presented as median (interquartile range); $p$-values are for comparison with the control group. Supplemental Table 1B: Viscoelastometric CT results from the control group and the anticoagulant groups, with pooling of the FXa inhibitors. Data are presented as median (interquartile range); $\mathrm{p}$-values are for comparison with the control group.

\section{Acknowledgements}

We would like to thank Alexander Chouker and his team for the support in the laboratories, as well as Ken Sutor for English editing support. Furthermore, we want to thank all involved medical staff working on the wards for their organizational support.

\section{Authors' contributions}

All authors contributed to the study conception and design. Material preparation, data collection and analysis were performed by Simon T. Schäfer, Philipp Groene, Daniela Wagner, and Tobias Kammerer. The first draft of the manuscript was written by Philipp Groene and Simon T. Schäfer and all authors commented on previous versions of the manuscript. All authors read and approved the final manuscript.

\section{Funding}

Thromboelastometric machines and reagents were provided by enicor $\mathrm{GmbH}$ (Munich, Germany). The funding sponsors enicor $\mathrm{GmbH}$ had no role in the collection, analysis or interpretation of data; in the writing of the manuscript, or in the decision to publish the results. Open Access funding enabled and organized by Projekt DEAL.

Availability of data and materials

The datasets used and/or analyzed during the current study are available from the corresponding author on reasonable request.

\section{Declarations}

\section{Ethics approval and consent to participate}

The protocol was approved by the Ludwig-Maximilians-University ethics committee (No 17-525-4). Written informed consent was obtained from all participants before they undertook any of the study procedures.

\section{Consent for publication}

Not applicable.

\section{Competing interests}

S.T.S. received lecture fees by CSL Behring, TEM international and Werfen as well as research grants by Octapharma and TEM International. P.G. received lecture fees by CSL Behring, D.W., T.K., L.K., S.M. and A.G. declare no conflict of interest.

\section{Author details}

'Department of Anaesthesiology, University Hospital Munich, LMU Munich, Marchioninistraße 15, 81377 Munich, Germany. ${ }^{2}$ Department of Anaesthesiology and Intensive Care Medicine, University Hospital of Cologne, Cologne, Germany. ${ }^{3}$ Department of Neurology, University Hospital Munich, LMU Munich, Munich, Germany. ${ }^{4}$ Department of Transfusion Medicine and Hemostaseology, University Hospital Augsburg, Augsburg, Germany.

${ }^{5}$ Department of Internal Medicine I - Cardiology, University Hospital Munich, LMU Munich, Munich, Germany.

Received: 24 November 2020 Accepted: 1 March 2021

Published online: 16 March 2021

References

1. Larson EA, German DM, Shatzel J, DeLoughery TG. Anticoagulation in the cardiac patient: a concise review. Eur J Haematol. 2019;102(1):3-19.

2. Proietti M, Lip GYH. Atrial fibrillation and stroke: making sense of recent observations on anticoagulation. Cardiol Clin. 2016;34(2):317-28.

3. Van Der Meersch $H$, De Bacquer D, De Vriese AS. Vitamin $K$ antagonists for stroke prevention in hemodialysis patients with atrial fibrillation: a systematic review and meta-analysis. Am Heart J. 2017;184:37-46.

4. Dahal K, Kunwar S, Rijal J, Schulman P, Lee J. Stroke, major bleeding, and mortality outcomes in warfarin users with atrial fibrillation and chronic kidney disease: a meta-analysis of observational studies. Chest. 2016;149(4): 951-9.

5. Kuruvilla M, Gurk-Turner C. A review of warfarin dosing and monitoring. Proc (Bayl Univ Med Cent). 2001;14(3):305-6.

6. Gressenberger P. Reversal strategies in patients treated with direct oral anticoagulants. Vasa. 2019;48(5):389-92.

7. Lindhoff-Last E. Direct oral anticoagulants (DOAC) - management of emergency situations. Hamostaseologie. 2017;37(4):257-66.

8. Levy JH, Tanaka KA, Dietrich W. Perioperative hemostatic management of patients treated with vitamin K antagonists. Anesthesiology. 2008;109(5): 918-26.

9. Thomas S, Makris M. The reversal of anticoagulation in clinical practice. Clin Med (Lond). 2018;18(4):314-9. 
10. Rawal A, Ardeshna D, Minhas S, Cave B, Ibeguogu U, Khouzam R. Current status of oral anticoagulant reversal strategies: a review. Ann Transl Med. 2019;7(17):411

11. Schmidt DE, Holmström M, Majeed A, Näslin D, Wallén H, Ågren A. Detection of elevated INR by thromboelastometry and thromboelastography in warfarin treated patients and healthy controls. Thromb Res. 2015;135(5):1007-11.

12. Favaloro EJ, Lippi G, Koutts J. Laboratory testing of anticoagulants: the present and the future. Pathology. 2011;43(7):682-92.

13. Henskens YMC, Gulpen AJW, van Oerle R, Wetzels R, Verhezen P, Spronk H, et al. Detecting clinically relevant rivaroxaban or dabigatran levels by routine coagulation tests or thromboelastography in a cohort of patients with atrial fibrillation. Thromb J. 2018;16:3.

14. Seyve L, Richarme C, Polack B, Marlu R. Impact of four direct oral anticoagulants on rotational thromboelastometry (ROTEM). Int J Lab Hematol. 2018;40(1):84-93.

15. Dias JD, Lopez-Espina CG, Ippolito J, Hsiao LH, Zaman F, Muresan AA, et al. Rapid point-of-care detection and classification of direct-acting oral anticoagulants with the TEG 6s: implications for trauma and acute care surgery. J Trauma Acute Care Surg. 2019;87(2):364-70.

16. Ebner M, Birschmann I, Peter A, Härtig F, Spencer C, Kuhn J, et al. Limitations of specific coagulation tests for direct oral anticoagulants: a critical analysis. J Am Heart Assoc. 2018;7(19):e009807.

17. Calatzis A, Wittwer M, Leyser H, Hipp Q, Spannagl M. ClotPro - a new generation viscoelastic whole blood coagulation analyzer. Hämostaseologie. 2018;4a:A32 [P 013].

18. Hartmann J, Murphy M, Dias JD. Viscoelastic hemostatic assays: moving from the laboratory to the site of care - a review of established and emerging technologies. Diagnostics (Basel). 2020;10(2):118.

19. Schäfer ST, Wiederkehr T, Kammerer T, Acevedo A-C, Feil K, Kellert L, et al. Real-time detection and differentiation of direct oral anticoagulants (rivaroxaban and dabigatran) using modified thromboelastometric reagents. Thromb Res. 2020:190:103-11.

20. Dias JD, Norem K, Doorneweerd DD, Thurer RL, Popovsky MA, Omert LA. Use of thromboelastography (TEG) for detection of new oral anticoagulants. Arch Pathol Lab Med. 2015;139(5):665-73.

21. Ten Cate H, Henskens YM, Lancé MD. Practical guidance on the use of laboratory testing in the management of bleeding in patients receiving direct oral anticoagulants. Vasc Health Risk Manag. 2017;13:457-67.

22. Oberladstätter D, Voelckel W, Schlimp C, Zipperle J, Ziegler B, Grottke O, et al. A prospective observational study of the rapid detection of clinicallyrelevant plasma direct oral anticoagulant levels following acute traumatic injury. Anaesthesia. 2020. https://doi.org/10.1111/anae.15254 [Online ahead of print].

23. Almarshad F, Alaklabi A, Bakhsh E, Pathan A, Almegren M. Use of direct oral anticoagulants in daily practice. Am J Blood Res. 2018;8(4):57-72.

24. Gosselin RC, Adcock DM, Douxfils J. An update on laboratory assessment for direct oral anticoagulants (DOACs). Int J Lab Hematol. 2019:41(Suppl 1):339 .

25. Pengo V, Denas G. Optimizing quality care for the oral vitamin $\mathrm{K}$ antagonists (VKAs). Hematology Am Soc Hematol Educ Program. 2018, 2018(1):332-8.

26. Smythe MA, Priziola J, Dobesh PP, Wirth D, Cuker A, Wittkowsky AK. Guidance for the practical management of the heparin anticoagulants in the treatment of venous thromboembolism. J Thromb Thrombolysis. 2016 $41(1): 165-86$.

\section{Publisher's Note}

Springer Nature remains neutral with regard to jurisdictional claims in published maps and institutional affiliations.

Ready to submit your research? Choose BMC and benefit from:

- fast, convenient online submission

- thorough peer review by experienced researchers in your field

- rapid publication on acceptance

- support for research data, including large and complex data types

- gold Open Access which fosters wider collaboration and increased citations

- maximum visibility for your research: over $100 \mathrm{M}$ website views per year

At BMC, research is always in progress.

Learn more biomedcentral.com/submissions 